\title{
Paravertebral catheter versus EPidural analgesia in Minimally invasive Esophageal resectioN: a randomized controlled multicenter trial (PEPMEN trial)
}

B. F. Kingma ${ }^{*}$, W. J. Eshuis ${ }^{2}$, E. M. de Groot ${ }^{1}$, M. L. Feenstra², J. P. Ruurda' ${ }^{1}$ S. S. Gisbertz², W. ten Hoope ${ }^{3}$, M. Marsman ${ }^{4}$, J. Hermanides ${ }^{3}$, M. W. Hollmann³ , C. J. Kalkman, M. D. P. Luyer ${ }^{5}$, G. A. P. Nieuwenhuijzen ${ }^{5}$, H. J. Scholten ${ }^{6}$, M. Buise ${ }^{6}$, M. J. van Det ${ }^{7}$, E. A. Kouwenhoven ${ }^{7}$, F. van der Meer ${ }^{8}$, G. W. J. Frederix ${ }^{9}$, E. Cheong ${ }^{10}$, K. al Naimi ${ }^{11}$, M. I. van Berge Henegouwen ${ }^{2+}$ and R. van Hillegersberg ${ }^{1 *+}$

\begin{abstract}
Background: Thoracic epidural analgesia is the standard postoperative pain management strategy in esophageal cancer surgery. However, paravertebral block analgesia may achieve comparable pain control while inducing less side effects, which may be beneficial for postoperative recovery. This study primarily aims to compare the postoperative quality of recovery between paravertebral catheter versus thoracic epidural analgesia in patients undergoing minimally invasive esophagectomy.
\end{abstract}

Methods: This study represents a randomized controlled superiority trial. A total of 192 patients will be randomized in 4 Dutch high-volume centers for esophageal cancer surgery. Patients are eligible for inclusion if they are at least 18 years old, able to provide written informed consent and complete questionnaires in Dutch, scheduled to undergo minimally invasive esophagectomy with two-field lymphadenectomy and an intrathoracic anastomosis, and have no contra-indications to either epidural or paravertebral analgesia. The primary outcome is the quality of postoperative recovery, as measured by the Quality of Recovery-40 (QoR-40) questionnaire on the morning of postoperative day 3. Secondary outcomes include the QoR-40 questionnaire score Area Under the Curve on postoperative days 1-3, the integrated pain and systemic opioid score and patient satisfaction and pain experience according to the International Pain Outcomes (IPO) questionnaire, and cost-effectiveness. Furthermore, the groups will be compared regarding the need for additional rescue medication on postoperative days $0-3$, technical failure of the pain treatment, duration of anesthesia, duration of surgery, total postoperative fluid administration day 0-3, postoperative vasopressor and inotrope use, length of urinary catheter use, length of hospital stay, postoperative complications, chronic pain at six months after surgery, and other adverse effects.

Discussion: In this study, it is hypothesized that paravertebral analgesia achieves comparable pain control while causing less side-effects such as hypotension when compared to epidural analgesia, leading to shorter postoperative length of stay on a monitored ward and superior quality of recovery. If this hypothesis is confirmed, the results of this study can be used to update the relevant guidelines on postoperative pain management for patients undergoing minimally invasive esophagectomy.

Trial registration: Netherlands Trial Registry, NL8037. Registered 19 September 2019.

\footnotetext{
*Correspondence: b.f.kingma@umcutrecht.nl;

r.vanhillegersberg@umcutrecht.nl

${ }^{\dagger}$ M. I. van Berge Henegouwen and R. van Hillegersberg contributed equally

to this work.

'Department of Surgery, University Medical Center Utrecht, POBOX 85500,

3508 GA Utrecht, The Netherlands

Full list of author information is available at the end of the article
}

(c) The Author(s). 2020 Open Access This article is distributed under the terms of the Creative Commons Attribution 4.0 International License (http://creativecommons.org/licenses/by/4.0/), which permits unrestricted use, distribution, and reproduction in any medium, provided you give appropriate credit to the original author(s) and the source, provide a link to the Creative Commons license, and indicate if changes were made. The Creative Commons Public Domain Dedication waiver (http://creativecommons.org/publicdomain/zero/1.0/) applies to the data made available in this article, unless otherwise stated. 


\section{Background}

Esophageal cancer is the 9th most common cancer worldwide and is increasingly diagnosed in the Western world, mainly due to the growing incidence of adenocarcinoma [1]. Esophagectomy is the core of curative treatment for esophageal cancer, achieving a 5-year survival rate of $40-50 \%$ when preceded by neoadjuvant therapy $[2,3]$. Traditional open transthoracic esophagectomy is associated with substantial postoperative thoracic pain, mainly due to the large intercostal incision, which may lead to decreased mobility, (pulmonary) complications, and delayed recovery [4]. In this light, adequate pain management is essential during the early postoperative phase. Thoracic epidural analgesia is the current gold standard for pain control in this context, as it was shown to be superior to systemic opioids in terms of pain control and pulmonary complications after open esophagectomy $[5,6]$. However, over the last decades, minimally invasive esophagectomy (MIE) is increasingly adopted, which is associated with less postoperative pain when compared to open surgery [7-9]. Furthermore, enhanced recovery protocols have been introduced for esophagectomy, aiming at fast mobilization and recovery after MIE [10-12]. As potential adverse effects of epidural analgesia include failed catheter placement, postoperative hypotension, and diminished mobilization, epidural analgesia may be counterproductive in achieving some of the key aims of enhanced recovery protocols for MIE $[13,14]$. Moreover, epidural analgesia is associated with severe neurological complications such as epidural hematoma or abscess formation in up to 1 in 1000 patients, which should be considered a serious issue $[15,16]$. Therefore, the value of epidural analgesia for MIE needs to be reconsidered in a state-of-the-art treatment setting.

In previous systematic reviews that included patients undergoing a variety of thoracotomy or thoracoscopy procedures (mostly lung surgery), paravertebral analgesia was found to achieve comparable pain relief while inducing less postoperative hypotension, urinary retention, and nausea when compared to epidural analgesia $[17,18]$. While these results seem to represent rather solid evidence in favour of paravertebral analgesia for thoracic surgery in general, transthoracic esophagectomy includes an abdominal phase in addition to the thoracic procedure. This means that results from studies that primarily included patients undergoing only thoracic surgery may not be generalizable to the setting of transthoracic esophagectomy. The hypothesized advantages of paravertebral analgesia over epidural analgesia were previously explored in systematic reviews of patients undergoing esophagectomy, which revealed that paravertebral analgesia achieves comparable pain control and possibly causes less hypotensive events [19, 20]. However, both reviews highlighted the lack of high-quality prospective studies in patients undergoing MIE [19, 20].
Most available studies on the efficacy of paravertebral analgesia after esophagectomy are retrospective in nature and included patients who underwent resection by an open approach, implying a substantial risk of bias and the possibility that these results cannot be generalized to the setting of MIE. Therefore, the primary aim of the current study is to compare the postoperative quality of recovery between paravertebral and epidural analgesia in patients undergoing MIE. Secondary objectives are to compare the efficacy, side-effects, and cost-effectiveness between these analgesic modalities. The hypothesis of this study is that paravertebral analgesia achieves comparable pain control while causing less side-effects, leading to shorter length of stay on a monitored ward and superior postoperative quality of recovery compared to epidural analgesia.

\section{Methods \\ Design}

This study represents a multicenter randomized superiority controlled trial that is conducted in 4 Dutch highvolume centers for esophageal cancer surgery: (1) University Medical Center Utrecht, (2) Amsterdam UMC (3), Catharina Hospital Eindhoven, and (4) Hospital Group Twente Almelo. The current version of the study protocol (V1.2) was approved by the Medical Ethical Committee of the University Medical Center Utrecht (reference number 19-588) and was prospectively registered in the Netherlands Trial Registry (trial number NL8037). Amendments to the protocol will first be presented to the ethics board and the approved adjustments are then also processed in the Netherlands Trial Registry. As this study is considered to carry negligible risks for participating patients, a data monitoring committee (DMC) was judged to be redundant.

\section{Patient population}

Patients who are scheduled to undergo elective minimally invasive esophagectomy with two-field lymphadenectomy, gastric conduit reconstruction, and intrathoracic anastomosis (i.e. Ivor Lewis procedure) are eligible for participation and will be included according to the flowchart in Fig. 1. Exclusion criteria are severe comorbidity (ASA score > III), coagulation disorders that prohibit epidural analgesia according to the Dutch Society for Anesthesiology guidelines "Neuraxisblock and anti-coagulation", other contraindications for epidural analgesia (e.g. local skin infection), allergy to local anesthetics, chronic opioid use prior to esophagectomy ( $>3$ months prior to the day of surgery), renal failure (eGFR $<50 \mathrm{~mL} / \mathrm{min}$.), inability to provide informed consent or complete questionnaires in Dutch, cervical lymph node dissection, and pregnancy. 


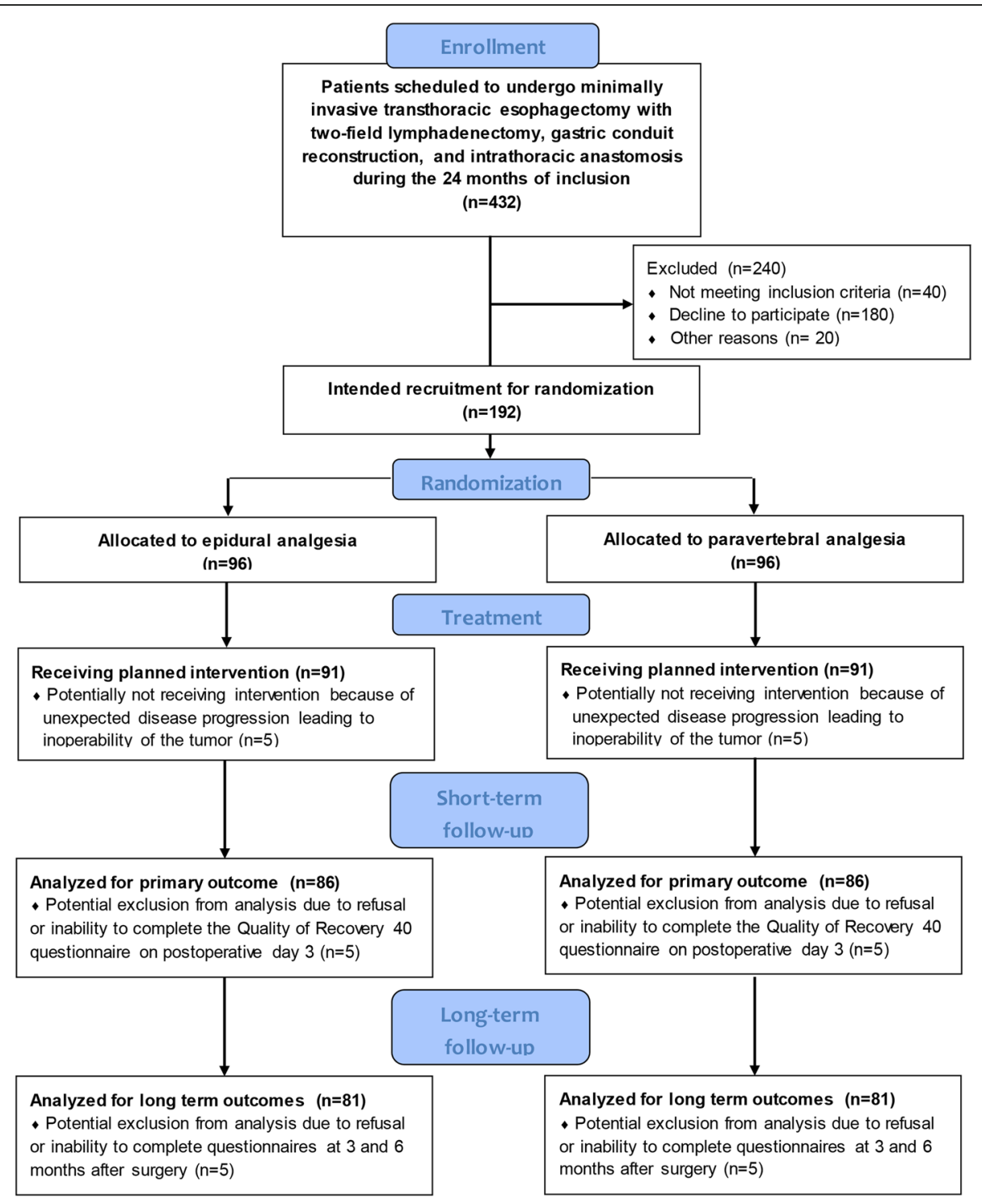

Fig. 1 PEPMEN inclusion flowchart

\section{Allocation to the treatment arms}

The coordinating investigators and involved trial nurses will screen patients for eligibility and obtain informed consent during patients' visits to the preoperative outpatient clinic. After inclusion and ultimately the day before surgery, one of the coordinating investigators or trial nurses will enter the patient in Castor EDC, which is the primary data capturing platform for this study and allows digital randomization. Randomization will be performed in a 1:1 ratio and stratified per including center to minimize the risk of center bias. The coordinating investigators of the study will ensure that patients receive the allocated analgesic modality at the time of esophagectomy. As a sham epidural catheterization would need to be performed prior to the induction of general anesthesia in the paravertebral group, which is considered unethical, patients will not be blinded. Health care providers will also not be blinded, since blinding would likely increase the risk of errors in pain management to an unacceptable extent.

\section{Surgical technique}

All patients will undergo MIE with two-field lymphadenectomy, gastric conduit reconstruction, and an intrathoracic anastomosis. The operation may be performed by conventional or robot-assisted thoracolaparoscopic surgery. The procedure starts with an abdominal laparoscopic phase, which involves mobilization of the stomach, abdominal lymphadenectomy and gastric conduit construction. Then, the patient will be placed in (semi) prone position for thoracoscopy to mobilize the esophagus and perform a mediastinal lymphadenectomy. One 
of the trocar ports is widened to a mini-thoracotomy for specimen extraction and when indicated, for the creation of the anastomosis. Gastrointestinal continuity is finally restored by a stapled or hand-sewn intrathoracic esophagogastric anastomosis. A maximum of one thoracic drain is placed on each side.

\section{Paravertebral analgesia (intervention)}

At the start of the thoracic phase of MIE, the surgeon places a paravertebral catheter at level T4-5 under thoracoscopic vision. After administering an initial bolus of $20 \mathrm{~mL}$ of bupivacaine $0.125 \%$, continuous infusion of bupivacaine $0.125 \%$ is started and postoperatively continued at an infusion rate of $8-12 \mathrm{~mL} /$ hour, depending on the patient's weight and comfort. Patient-controlled intravenous opioid analgesia is additionally provided according to the local protocol in each center.

\section{Epidural analgesia (control)}

An epidural catheter is placed by the anesthesiologist at an intervertebral level between T5 and T8 prior to the induction of general anesthesia. After induction, a bolus of $5-10 \mathrm{~mL}$ of bupivacaine $0.25 \%$ is administered and traditional continuous epidural analgesia is initiated (bupivacaine $0.125 \%$ + sufentanil $0.5 \mathrm{mcg} / \mathrm{ml}$ ) in a dose of 6-14 mL/hour, depending on the patient's weight and comfort. Patient-controlled intravenous opioid analgesia is provided as escape medication according to the local protocol in each center.

\section{Outcome measures}

The primary outcome measure is the total score on the Quality of Recovery 40 (QoR-40 [21]) questionnaire on the morning of postoperative day 3. The QoR-40 is a validated composite endpoint that can be used to evaluate analgesic modalities for postoperative pain control [22]. The main secondary outcome measures include the QoR-40 scores on postoperative days 1-2, the patient's perception of postoperative pain management on days 1-3 (International Pain Outcomes (IPO) questionnaire), the need for escape pain medication on days 1-3, additional opioid consumption on days 1-3, technical complications, analgesia related side-effects, need for inotropic and vasopressive medication, length of stay on a monitored unit and in the hospital, postoperative complications, quality of life at 3 and 6 months after surgery, pain at 3 and 6 months after surgery (VAS score), and cost-effectiveness.

\section{Data collection}

All data will be collected and stored in Castor EDC (https://www.castoredc.com) and the coordinating investigators will oversee the overall data collection process. Castor EDC generates a subject number for each patient and securely stores all entered research data in a pseudonymized fashion. A code file that links subjects numbers to individual patients will be securely stored in each center, and will only be accessible to authorized study staff. Collected baseline data (age, gender, body mass index, comorbidities), treatment details (neoadjuvant therapy, surgical techniques, postoperative complications, mortality), and data regarding blood pressure (i.e. vasopressor and inotropic use, fluid administration, weight) will be prospectively entered in a case report form with built-in validation checks. Patient-reported outcome measures (PROMs) will be collected during the postoperative hospitalization by asking patients to complete the QoR-40 and IPO questionnaires on postoperative days 1, 2, and 3. Furthermore, Quality of life questionnaires (EORTC QLQ-C30 and QLQ-OG25) will be sent to patients before surgery, at 3 months followup, and at 6 months follow-up after esophagectomy. Pain scores (VAS) at 3 and 6 months after esophagectomy will be collected and registered during the patients' regular outpatient follow-up visits. The timeline of the study procedures is summarized in Fig. 2.

\section{Quality control}

Data collection and management are centrally monitored in all centers. The independent monitor of the study will visit each participating center at the start of inclusion, after 1 year of inclusion, and at the end of inclusion. All paravertebral procedures performed during the study will be recorded and stored for quality control. Serious adverse events related to the study procedures will be reported to the sponsor of the study without any undue delay.

\section{Sample size}

The QoR-40 questionnaire was used as the primary outcome measure to calculate a sample size based on superiority of paravertebral analgesia when compared to epidural analgesia [21]. Backward and forward translations of the QoR-40 were validated by the study consortium to ensure the accuracy of the Dutch version. Based on previous literature, a QoR-40 score of 182 points with a standard deviation of 14 on postoperative day 3 is expected in the control group [23]. A difference of 6 points on the QoR-40 was assumed to indicate a minimally clinically relevant difference based on literature [24]. Hence, a total sample size of 172 patients is required (86 patients per group) to detect this difference with a statistical power of $80 \%$ and a significance level of (alpha) $P<$ 0.05. Anticipating a $10 \%$ loss to follow up, 192 patients will be included. Only patients who withdraw before surgery will be replaced by new subjects. 


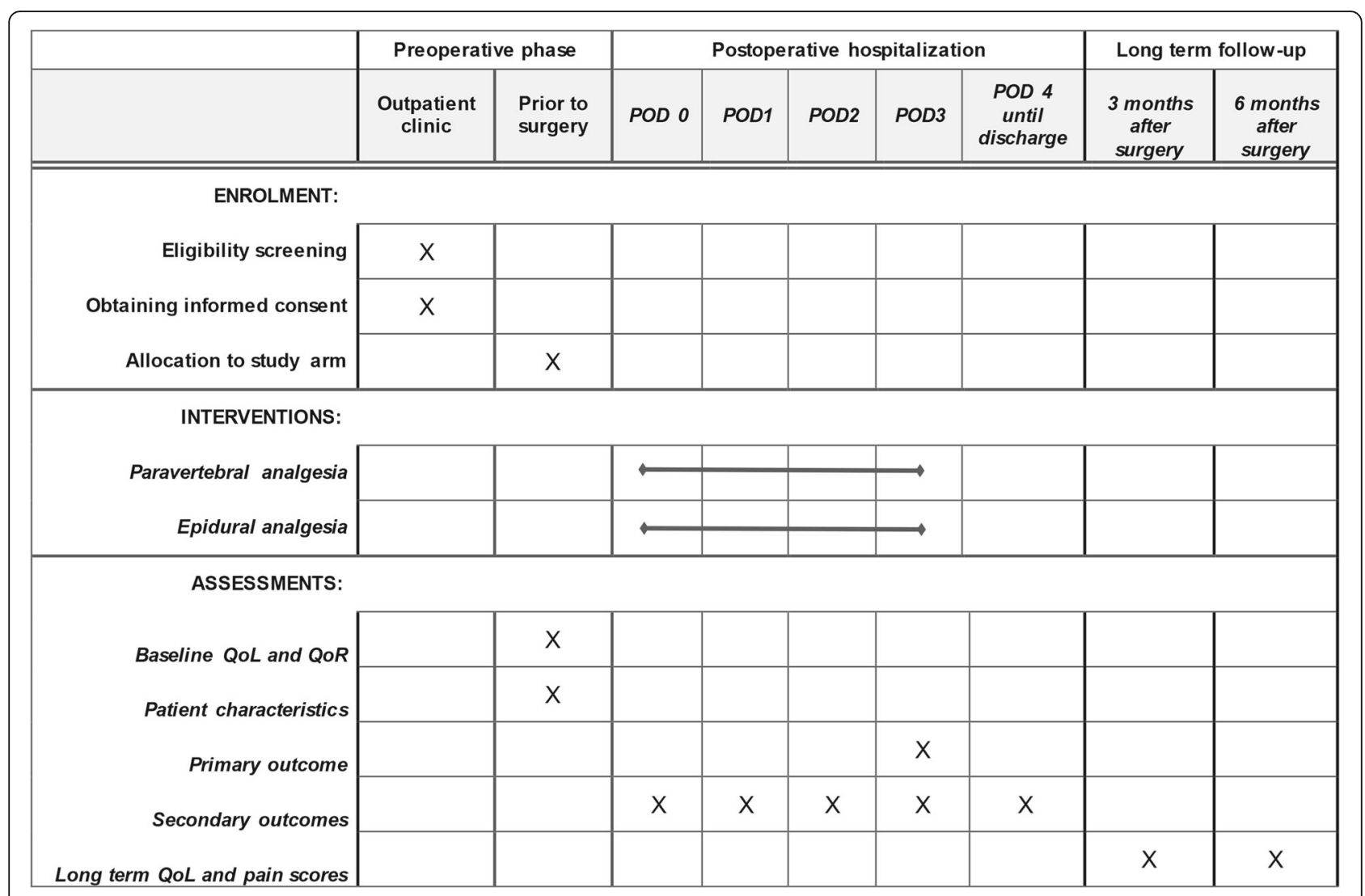

Legend: POD; postoperative day. POD 0 refers to the day of surgery. QoL; quality of life as measured by the EORTC C30 and OG-25 questionnaires QoR; quality of recovery as measured by the QoR-40 questionnaire.

Fig. 2 PEPMEN timeline of study procedures

\section{Statistical analyses}

The difference in primary outcome between the two groups will be compared using an independent t-test based on the intention-to-treat principle. The primary outcome will be analyzed with a general linear model for continuous outcomes. In the analysis, the preoperative (baseline) QoR-40 measurement, age, and comorbidities will be included as potential confounders. Validity of the model (normality, homoscedasticity) will be assessed by means of residue analysis. A per-protocol analysis will additionally be performed in the same manner. Analyses of the secondary outcomes are performed depending on data type and distribution. Chi-square tests will be performed for categorical data. The t-test or Mann-Whitney $\mathrm{U}$ test will be used to compare continuous outcomes with a normal or non-normal distribution, respectively.

\section{Time schedule}

The study has started on December 3rd 2019. The first 24 months will be used for patient inclusion. After finishing patient inclusion, there will be 6 months of follow-up and an additional 6 months of data analysis.

\section{Discussion}

Thoracic epidural analgesia has long been the gold standard for pain management following esophagectomy. However, paravertebral analgesia is increasingly suggested as a good alternative for patients undergoing MIE, as the catheter can be placed under direct thoracoscopic vision possibly reduces the incidence of hypotensive events. Furthermore, by avoiding epidural catheterization and its potentially severe neurological complications, paravertebral analgesia may be safer. However, the currently available studies mainly included patients undergoing other thoracic surgical procedures and high-quality prospective studies investigating paravertebral analgesia for esophageal cancer surgery are lacking. Therefore, the PEPMEN study aims to compare paravertebral analgesia versus epidural analgesia in patients undergoing MIE by means of a prospective multicenter randomized controlled trial.

Adequate analgesia is one of the key parts of enhanced recovery after surgery (ERAS) protocols for major surgical procedures, aiming to achieve effective pain control whilst avoiding the side-effects of high doses of systemic opioids. These aims were also described in the recently developed ERAS Society guidelines for esophagectomy, 
which state that regional analgesia should be the backbone of pain management in enhanced recovery after esophagectomy protocols [25]. In absence of trials in patients undergoing MIE, both epidural and paravertebral analgesia were considered to be suitable options based on extrapolated evidence, although no recommendations in favour of either modality could be made [25]. Retrospective cases series suggest that paravertebral analgesia is associated with less technical failure and reduces the incidence of hypotensive events, which may promote postoperative recovery $[26,27]$. Combined with inconclusive ERAS recommendations regarding the use of either epidural or paravertebral analgesia, these retrospective findings represent an important rationale to perform a prospective randomized controlled trial comparing paravertebral and epidural analgesia regarding perioperative outcomes and treatment costs in patients undergoing MIE.

In contrast to epidural analgesia, paravertebral analgesia can only induce a unilateral sensory block of thoracic dermatomes. Nonetheless, this unilateral thoracic block is expected to achieve adequate control of chest pain after MIE, especially when combined with patientcontrolled intravenous opioid analgesia as an escape for breakthrough discomfort [28]. If paravertebral analgesia in fact achieves adequate pain control, the avoidance of epidural-related side-effects likely facilitates fast recovery after MIE [29, 30], which is the primary interest of this study. Since postoperative recovery is multifactorial, a composite endpoint was considered to be most appropriate and therefore the QoR-40 questionnaire was chosen as primary outcome. The QoR-40 consists of 40 questions that are divided in separate sections that aim to evaluate the presence and extent of pain, symptoms, comfort, emotional well-being, physical independence, and satisfaction with treatment [21]. As the hypothesized advantages of paravertebral analgesia are expected to impact several of these sections, the composite QoR-40 score is considered to be a good parameter for postoperative recovery.

One might argue that the current randomized study design could be replaced by a prospective cohort study with sequential measurement of a control group followed by an intervention group which might be acceptable regarding the risk of selection bias, as the demographics of esophageal cancer patients are not expected to change within a period of a few years. However, it should be noted that the intraoperative techniques and perioperative facets of enhanced recovery protocols are continuously subjected to alterations based on new developments in the field of MIE. In case of a non-randomized sequential prospective design, changes in parts of the perioperative protocol during the study (e.g. the postoperative mobilization program) would not be possible without potentially inflicting a bias that significantly impacts the interpretability of the results. By choosing a randomized study design with stratification per center, the participating centers can still implement perioperative developments in other areas than the pain management regimen without compromising the reliability of the comparison between analgesic techniques.

In summary, the PEPMEN study is a randomized controlled multicenter trial comparing paravertebral versus epidural analgesia in patients undergoing MIE in 4 highvolume centers for esophageal cancer surgery in the Netherlands. The primary endpoint will be the quality of recovery, as measured by the QoR- 40 questionnaire on postoperative day 3 . By improving the quality of recovery and shortening the length of stay on the ICU, paravertebral analgesia is expected to reduce the costs of perioperative care for patients undergoing MIE. A total sample size of 192 patients is required and the duration of the study will be 3 years.

\section{Abbreviations \\ ASA: American Society of Anesthesiologists score; DMC: Data Monitoring Committee; eGFR: Estimated Glomerular Filtration Rate; ERAS: Enhanced Recovery After Surgery; IPO: International Pain Outcomes questionnaire; MIE: Minimally Invasive Esophagectomy; PROMs: Patient Reported Outcome Measures; QoR-40: Quality of Recovery 40 questionnaire; VAS: Visual Analog Scale}

\section{Acknowledgements}

Not applicable.

\section{Dissemination of results}

After completion of the study, the results will be published in a peer reviewed journal. A summary of the results will also be made publically available on the trial website (http://www.pepmentrial.nl).

\section{Authors' contributions}

The principal investigators of the trial are $\mathrm{RvH}$ and $\mathrm{MvBH}$. The study protocol was drafted by FK and WE. Surgical expertise was provided by JR, SG, ML,

$\mathrm{GN}, \mathrm{MvD}, \mathrm{EK}$, and EC. Anesthesiological expertise was gained from $\mathrm{MH}, \mathrm{CK}$, WtH, MM, JH, HS, MB, FvdM, and KaN. The cost-effectiveness analyses will be performed according to the methodology that was established by GF. The logistic arrangement for the trial were made by EdG and MF, who will act as the coordinating investigators during the trial. All authors read and approved the manuscript.

\section{Funding}

The Netherlands Organization for Health Research and Development (ZonMw) has peer reviewed this study and decided to support this study by means of a health efficiency grant (852002004). This funding body has no role in the collection, analyses or interpretation of data, nor will it be involved in the writing of the manuscript. These tasks will be fulfilled by the coordinating investigators (EdG and MF) on behalf of the PEPMEN consortium.

\section{Availability of data and materials}

The datasets of this study will be available from the corresponding author on reasonable request after completion.

\section{Ethics approval and consent to participate}

As this study falls under the Medical Research Involving Human Subjects Act (WMO), ethical approval was requested and gained from the Medical Ethical Committee of the University Medical Center Utrecht (reference number 19588). Written informed consent to participate will be obtained from all study participants. 


\section{Consent for publication}

Not applicable.

\section{Competing interests}

The authors declare that they have no competing interests.

\section{Author details}

'Department of Surgery, University Medical Center Utrecht, POBOX 85500, 3508 GA Utrecht, The Netherlands. ${ }^{2}$ Department of Surgery, Amsterdam UMC, Amsterdam, The Netherlands. ${ }^{3}$ Department of Anesthesiology, Amsterdam UMC, Amsterdam, The Netherlands. ${ }^{4}$ Department of Anesthesiology, University Medical Center Utrecht, Utrecht, The Netherlands. ${ }^{5}$ Department of Surgery, Catharina Hospital, Eindhoven, The Netherlands. ${ }^{6}$ Department of Anesthesiology, Catharina Hospital Eindhoven, Eindhoven, The Netherlands. ${ }^{7}$ Department of Surgery, Hospital Group Twente, Almelo, The Netherlands. ${ }^{8}$ Department of Anesthesiology, Hospital Group Twente Almelo, Almelo, The Netherlands. ${ }^{9}$ Department of Public Health, Healthcare Innovation \& Evaluation and Medical Humanities, University Medical Center Utrecht, Utrecht, the Netherlands. ${ }^{10}$ Department of Surgery, Norfolk and Norwich University Hospital, Norwich, UK. "'Department of Anesthesiology, Norfolk and Norwich University Hospital, Norwich, UK.

Received: 3 January 2020 Accepted: 29 January 2020

Published online: 22 February 2020

\section{References}

1. Bray F, Ferlay J, Soerjomataram I, Siegel RL, Torre LA, Jemal A. Global cancer statistics 2018: GLOBOCAN estimates of incidence and mortality worldwide for 36 cancers in 185 countries. CA Cancer J Clin. 2018;68(6):394-424.

2. Shapiro J, van Lanschot JJB, Hulshof MCCM, van Hagen P, van Berge Henegouwen MI, Wijnhoven BPL, et al. Neoadjuvant chemoradiotherapy plus surgery versus surgery alone for oesophageal or junctional cancer (CROSS): long-term results of a randomised controlled trial. Lancet Oncol. 2015;16(9):1090-8.

3. van der Sluis PC, Ruurda JP, Verhage R, van der Horst S, Haverkamp L, Siersema PD, et al. Oncologic long-term results of robot-assisted minimally invasive Thoracolaparoscopic Esophagectomy with two-field lymphadenectomy for esophageal Cancer. Ann Surg Oncol. 2015;22(Suppl 3):S1350-6.

4. Richardson J, Sabanathan S, Mearns AJ, Evans CS, Bembridge J, Fairbrass M. Efficacy of pre-emptive analgesia and continuous extrapleural intercostal nerve block on post-thoracotomy pain and pulmonary mechanics. J Cardiovasc Surg. 1994;35(3):219-28.

5. Flisberg $P$, Tornebrandt $K$, Walther $B$, Lundberg J. Pain relief after esophagectomy: thoracic epidural analgesia is better than parenteral opioids. J Cardiothorac Vasc Anesth. 2001;15(3):282-7.

6. Cense HA, Lagarde SM, de Jong K, Omloo JM, Busch OR, Henny C, et al. Association of no epidural analgesia with postoperative morbidity and mortality after transthoracic esophageal cancer resection. J Am Coll Surg. 2006;202(3):395-400.

7. Haverkamp L, Seesing MF, Ruurda JP, Boone J, V Hillegersberg R. Worldwide trends in surgical techniques in the treatment of esophageal and gastroesophageal junction cancer. Dis Esophagus. 2017;30(1):1-7.

8. Biere SS, van Berge Henegouwen MI, Maas KW, Bonavina L, Rosman C, Garcia JR, et al. Minimally invasive versus open oesophagectomy for patients with oesophageal cancer: a multicentre, open-label, randomised controlled trial. Lancet. 2012;379(9829):1887-92.

9. van der Sluis PC, van der Horst S, May AM, Schippers C, Brosens LAA, Joore HCA, et al. Robot-assisted minimally invasive Thoracolaparoscopic Esophagectomy versus open transthoracic Esophagectomy for Resectable esophageal Cancer: a randomized controlled trial. Ann Surg. 2019;269(4):621-30.

10. Markar SR, Schmidt H, Kunz S, Bodnar A, Hubka M, Low DE. Evolution of standardized clinical pathways: refining multidisciplinary care and process to improve outcomes of the surgical treatment of esophageal cancer. J Gastrointest Surg. 2014;18(7):1238-46.

11. Markar SR, Karthikesalingam A, Low DE. Enhanced recovery pathways lead to an improvement in postoperative outcomes following esophagectomy: systematic review and pooled analysis. Dis Esophagus. 2015;28(5):468-75.

12. Schmidt HM, El Lakis MA, Markar SR, Hubka M, Low DE. Accelerated recovery within standardized recovery pathways after Esophagectomy: a prospective cohort study assessing the effects of early discharge on outcomes, readmissions, patient satisfaction, and costs. Ann Thorac Surg. 2016;102(3):931-9.
13. Kingma BF, Visser E, Marsman M, Ruurda JP, van Hillegersberg R. Epidural analgesia after minimally invasive esophagectomy: efficacy and complication profile. Dis Esophagus. 2019;32(8). https://doi.org/10.1093/ dote/doy 116.

14. Hermanides J, Hollmann MW, Stevens MF, Lirk P. Failed epidural: causes and management. Br J Anaesth. 2012;109(2):144-54.

15. Kooij FO, Schlack WS, Preckel B, Hollmann MW. Does regional analgesia for major surgery improve outcome? Focus on epidural analgesia. Anesth Analg. 2014;119(3):740-4.

16. Bos EME, Haumann J, de Quelerij M, Vandertop WP, Kalkman CJ, Hollmann MW, et al. Haematoma and abscess after neuraxial anaesthesia: a review of 647 cases. Br J Anaesth. 2018;120(4):693-704.

17. Yeung JH, Gates S, Naidu BV, Wilson MJ, Gao SF. Paravertebral block versus thoracic epidural for patients undergoing thoracotomy. Cochrane Database Syst Rev. 2016;2:CD009121.

18. Davies RG, Myles PS, Graham JM. A comparison of the analgesic efficacy and side-effects of paravertebral vs epidural blockade for thoracotomy--a systematic review and meta-analysis of randomized trials. Br J Anaesth. 2006;96(4):418-26.

19. Visser E, Marsman M, van Rossum PSN, Cheong E, Al-Naimi K, van Klei WA, et al. Postoperative pain management after esophagectomy: a systematic review and meta-analysis. Dis Esophagus. 2017;30(10):1-11.

20. Hughes M, Yim I, Deans DAC, Couper GW, Lamb PJ, Skipworth RJE. Systematic review and meta-analysis of epidural analgesia versus different analgesic regimes following Oesophagogastric resection. World J Surg. 2018:42(1):204-10.

21. Gornall BF, Myles PS, Smith CL, Burke JA, Leslie K, Pereira MJ, et al. Measurement of quality of recovery using the QoR-40: a quantitative systematic review. Br J Anaesth. 2013;111(2):161-9.

22. Rothaug J, Zaslansky R, Schwenkglenks M, Komann M, Allvin R, Backstrom R, et al. Patients' perception of postoperative pain management: validation of the international pain outcomes (IPO) questionnaire. J Pain. 2013;14(11):1361-70.

23. Hansdottir V, Philip J, Olsen MF, Eduard C, Houltz E, Ricksten SE. Thoracic epidural versus intravenous patient-controlled analgesia after cardiac surgery: a randomized controlled trial on length of hospital stay and patient-perceived quality of recovery. Anesthesiology. 2006;104(1):142-51.

24. Myles PS, Myles DB, Galagher W, Chew C, MacDonald N, Dennis A. Minimal clinically important difference for three quality of recovery scales. Anesthesiology. 2016;125(1):39-45.

25. Low DE, Allum W, De Manzoni G, Ferri L, Immanuel A, Kuppusamy M, et al. Guidelines for perioperative Care in Esophagectomy: enhanced recovery after surgery (ERAS((R))) society recommendations. World J Surg. 2019;43(2):299-330.

26. Donohoe CL, Phillips AW, Flynn E, Donnison C, Taylor CL, Sinclair RCF, et al. Multimodal analgesia using intrathecal diamorphine, and paravertebral and rectus sheath catheters are as effective as thoracic epidural for analgesia post-open two-phase esophagectomy within an enhanced recovery program. Dis Esophagus. 2018;31(6). https://doi.org/10.1093/dote/doy006.

27. Phillips S, Dedic-Hagan J, Baxter DF, Van der Wall H, Falk GL. A novel technique of paravertebral thoracic and Preperitoneal analgesia enhances early recovery after Oesophagectomy. World J Surg. 2018;42(6):1787-91.

28. Zhang W, Fang C, Li J, Geng QT, Wang S, Kang F, et al. Single-dose, bilateral paravertebral block plus intravenous sufentanil analgesia in patients with esophageal cancer undergoing combined thoracoscopic-laparoscopic esophagectomy: a safe and effective alternative. J Cardiothorac Vasc Anesth. 2014;28(4):966-72.

29. van den Berg JW, Tabrett K, Cheong E. Paravertebral catheter analgesia for minimally invasive Ivor Lewis oesophagectomy. J Thorac Dis. 2019;11(Suppl 5):S786-93.

30. Cheong E. How minimally invasive esophagectomy was implemented at the Norfolk and Norwich University hospital. J Thorac Dis. 2017;9(Suppl 8): S879-85.

\section{Publisher's Note}

Springer Nature remains neutral with regard to jurisdictional claims in published maps and institutional affiliations. 